\title{
Synkope
}

\section{Harmlose Ohnmacht oder Vorbote des akuten Herztodes?}

Eine Synkope stellt den Arzt vor die Frage: Handelt es sich um eine harmlose orthostatische bzw. neurokardiogene Kreislaufregulationsstörung oder eine gefährliche bradykarde bzw. tachykarde Arrhythmie? Wie Sie mit relativ einfachen Mitteln, nämlich Anamnese, klinischer Untersuchung und EKG, zur richtigen Diagnose kommen können, erfahren Sie im folgenden Bericht.

— Eine Synkope ist ein plötzlich einsetzender, spontan reversibler Bewusstseins- und Tonusverlust infolge zerebraler Minderperfusion. Meist handelt es sich, so Prof. G. Fröhlig, Homburg/Saar, um eine harmlose orthostatische oder neurokardiogene Synkope mit sehr guter Prognose. Anders ist die Situation bei Patienten mit kardialer Grunderkrankung. Hier muss ein synkopales Ereignis immer als Vorbote des akuten Herztodes gesehen und sekundärpräventive Maßnahmen diskutiert werden.

\section{Was die Anamnese verrät}

Bei der Anamnese geht es zunächst darum, eine echte Synkope abzugrenzen von anderen Krankheitsbildern, die mit plötzlichem Bewusstseinsverlust einhergehen, z. B. Krampfanfall, Kataplexie, hypoglykämischer Schock oder Intoxikation. Die Familienanamnese kann auf eine angeborene elektrische Anomalie des Herzens hinweisen wie z. B. ein Brugada- oder ein Long-QT-Syndrom. Eine Zungenbissverletzung, ein Dämmerzustand oder Verwirrtheit nach dem Ereignis sprechen für einen Krampfanfall. Typisch für eine kardiale Ursache ist, dass die Bewusstlosigkeit ohne Vorboten eintritt. Patienten mit neurokardiogener oder orthostatischer Synkope berichten dagegen, dass sie vor dem Ereignis ein Leeregefühl im Kopf, Schwindel oder starkes Schwitzen verspürt haben.

\section{Auf Herzgeräusche achten}

Die Auskultation ist essenziell, um nicht eine Aortenstenose oder eine hypertrophe obstruktive Kardiomyopathie zu übersehen. Finden sich Zeichen ei- ner Herzinsuffizienz, besteht der Verdacht auf eine hämodynamisch wirksame Herzrhythmusstörung. Mithilfe der Blutdruckmessung in Ruhe und nach dem Aufstehen kann eine orthostatische Reaktion erfasst werden. Zur Basisdiagnostik gehört auch das RuheEKG. Dabei muss insbesondere auf Blockierungen, eine QT-Verlängerung und Zeichen der linksventrikulären Hypertrophie geachtet werden.

\section{Wann stationär abklären?}

Bei Patienten unter 45 Jahren ohne Familienanamnese für akuten Herztod und unauffälligem klinischen und EKGBefund ist im Allgemeinen keine weitere Diagnostik erforderlich. Eine stationäre Abklärung ist ratsam, wenn in der Familie ein akuter Herztod aufgetreten ist oder der Verdacht auf eine strukturelle Herzerkrankung besteht. Gleiches gilt, wenn die Synkope zu erheblichen Verletzungen geführt hat oder bei körperlicher Belastung aufgetreten ist.

\section{Farbdoppler-Echo bei}

\section{V. a. kardiale Genese}

Bei Verdacht auf kardiale Genese der Synkope sollte eine Farbdopplerechokardiografie durchgeführt werden. Damit können sowohl hämodynamisch wirksame Vitien als auch eine Herzinsuffizienz bzw. eine hypertrophe Kardiomyopathie nachgewiesen bzw. ausgeschlossen werden. Findet sich eine verminderte linksventrikuläre Pumpfunktion, ist eine Koronarangiografie indiziert, um eine diffuse KHK von einer dilatativen Kardiomyopathie abzugrenzen, so Prof. J. Brachmann, Coburg.

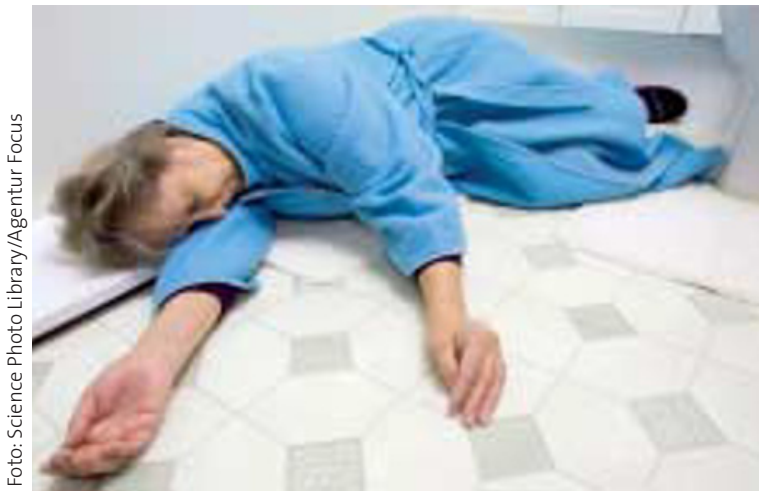

Bei kardialer Ursache tritt die Bewusstlosigkeit ohne „Vorwarnung“ ein.

\section{ICD zur Sekundärprävention?}

Bei Patienten mit kardialer Erkrankung muss immer eine maligne Herzrhythmusstörung als Ursache der Synkope angenommen und ein Langzeit-EKG durchgeführt werden. Doch auch damit lässt sich gerade bei herzinsuffizienten Patienten eine lebensbedrohliche Herzrhythmusstörung nicht mit letzter Sicherheit ausschließen. Deshalb sollte bei Personen mit hochgradig eingeschränkter linksventrikulärer Pumpfunktion nach Synkope die prophylaktische Implantation eines automatischen Defibrillators diskutiert werden.

Eine kardiale Synkope muss aber nicht durch eine Rhythmusstörung verursacht sein. Bei Patienten mit Aortenstenose, hypertropher obstruktiver Kardiomyopathie oder Lungenembolie kann auch eine akute hämodynamische Verschlechterung zu Bewusstlosigkeit führen. Deshalb empfiehlt sich bei Patienten mit höhergradiger Aortenstenose zunächst der prothetische Herzklappenersatz. Bei Patienten mit hypertropher obstruktiver Kardiomyopathie und zusätzlichen Risikofaktoren ist nach einer Synkope eine ICDImplantation erforderlich. STI *

- Quelle: 12. Dresdner Symposium „Herz und Gefäße" 